PROCEEDINGS OF THE

AMERICAN MATHEMATICAL SOCIETY

Volume 35, Number 2, October 1972

\title{
ON SUBFIELDS OF COUNTABLE CODIMENSION
}

\author{
A. BIAEYNICKI-BIRULA
}

\begin{abstract}
In [2] the authors asked if any two real closed subfields $R, R^{\prime}$ of the field of complex numbers $C$ such that $R(\sqrt{ }(-1))=$ $R^{\prime}(\sqrt{ }(-1))=C$ are isomorphic. It is not difficult to see that the answer is negative. This is proved in the first part of the note. In the second we study the problem if any field which is not prime contains a proper subfield of countable (finite or infinite) codimension.
\end{abstract}

1. Proposition 1. Let $R$ be any real closed field of power continuum. Then the field $R(\sqrt{ }(-1))$ is isomorphic to the field of complex numbers.

Proof. It is well known (see e.g. [3, p. 274]) that if the field $R$ is real closed then the field $R(\sqrt{ }(-1))$ is algebraically closed. Since any algebraically closed field of power continuum and of characteristic zero is isomorphic to the field of complex numbers hence if $R$ is real closed of power continuum then $R(\sqrt{ }(-1))$ is isomorphic to the field of complex numbers. Thus the proposition is proved.

Because there exist nonisomorphic real closed fields of power continuum (e.g. the field $R$ and the real closure of the ordered field $R(t)$, where $0<t<a$, for any positive $a \in R$ ) hence we obtain the following corollary which provides an answer to the question asked in $[2, \operatorname{Remark}(\mathrm{V}), \mathrm{p}$. 257].

COROLlary 1. There exist nonisomorphic real closed fields $R_{\mathbf{1}}, R_{\mathbf{2}}$ such that $R_{1}(\sqrt{ }(-1)), R_{2}(\sqrt{ }(-1))$ are isomorphic to the field of complex numbers.

2. It follows from Proposition 1 of [2] (as well as from the ArtinSchreier theorem [1, p. 316]) that no real closed field contains a proper subfield of finite codimension and the same property holds for all algebraically closed fields of finite characteristic. Intuitively this means that no proper subfield of those fields can be "very close" to the whole field. Therefore for a given field one may ask how close to the field can be the proper subfields of the field and the following conjecture seems to be reasonable (assuming the axiom of choice):

Any nonprime field contains a proper subfield of countable (finite or infinite) codimension.

Received by the editors October 15, 1971.

AMS 1970 subject classifications. Primary 12D99, 12 F99.

(c) American Mathematical Society 1972 
Though we are not able to prove (or disprove) this conjecture the following result may be considered as an evidence of its validity.

THEOREM 1. Let $L$ be a field. Then $L$ contains a proper subfield $K$ of countable codimension if one of the following conditions is satisfied:

(a) $L$ is algebraically closed;

(b) L is real closed;

(c) $L$ is a p-adic field $Q_{p}$ (i.e., completion of $Q$ in the $p$-adic topology).

First we state two simple lemmas.

Lemma.1. Let $G$ be a topological group and let $H_{1}, H_{2}$ be two subgroups of $G$. Let $G$ be generated by $H_{1} \cup H_{2}$ and let for any integer $n, H_{1}$ and $H_{2}$ contain only finitely many closed normal subgroups of index less than $n$. Then the same property holds for $G$.

Proof. It suffices to show that for any fixed finite group $G_{0}$ there exist only finitely many continuous homomorphisms $\varphi: G \rightarrow G_{0}$. Any such homomorphism is uniquely determined by its restriction $\varphi\left|H_{1}, \varphi\right| H_{2}$. Since $H_{1}, H_{2}$ contain only finitely many closed normal subgroups of index less than the order of $G_{0}$ hence there is only a finite number of homomorphisms $H_{1} \rightarrow G_{0}$ and $H_{2} \rightarrow G_{0}$. Thus the lemma is proved.

LeMma 2. Let $L_{1}$ be a Galois (possibly infinite) extension of $K_{1}$. Let $H$ be a subgroup of $\mathrm{Gal}\left(L_{1} / K_{1}\right)$. If for any integer $n, H$ contains only a finite number of closed normal subgroups of index less than $n$ then the subfield $L_{1}^{H}$ has countable codimension in $L_{1}$.

For the proof notice that it follows from the assumptions on $H$ (and from Galois theory) that for any integer $n$ there exists only a finite number of normal extensions of $L_{1}^{H}$ contained in $L_{1}$ and of degree less than $n$ over $L_{1}^{H}$.

Proof OF THE THEOREM. (a) Suppose that $L$ is algebraically closed. Let $L_{0}$ be the prime subfield of $L$ and let $\left\{x_{\alpha}\right\}$ be a transcendence basis of $L$ over $L_{0}$. Let $G=\operatorname{Gal}\left(L / L_{0}\left(\left\{x_{\alpha}\right\}\right)\right)$ and let $K_{1}=L^{G}$. Then $K_{1} \neq L$ and $L$ is a Galois extension of $K_{1}$. Hence there exists $a \in G, a \neq 1$. Let $H$ be the subgroup of $G$ generated by $a$. Then the subfield $K=L^{H}$ is proper of countable (by Lemma 2) codimension.

(b) Suppose that the field $L$ is real closed. Then $L_{1}=L(\sqrt{ }(-1))$ is algebraically closed. Let $Q$ be the prime subfield of $L$ and let $\left\{x_{\alpha}\right\}$ be a transcendence basis of $L$ over $Q$. Let $G=\operatorname{Gal}\left(L_{1} / Q\left(\left\{x_{\alpha}\right\}\right)\right)$. Then the conjugation $\sigma: L_{1} \rightarrow L_{1}$ over $L$ (i.e. $\sigma(a+b \sqrt{ }(-1))=a-b \sqrt{ }(-1)$, for any $a, b \in L$ ) belongs to $G$ and the subgroup $\{1, \sigma\}$ of $G$ is not normal.

In fact, one can easily see that $L$ is not a normal extension of $Q\left(\left\{x_{\alpha}\right\}\right)$ ( $L$ cannot contain all roots of all irreducible polynomials $f \in Q[x]$ of odd 
degree). Let $\sigma^{\prime}$ be a conjugate of $\sigma$ in $G$ different from $\sigma$ and let $H$ be the subgroup of $G$ generated by $\left\{\sigma, \sigma^{\prime}\right\}$. Then $L_{1}^{H} \subsetneq L$ and by Lemmas 1 and 2 , $L_{1}^{H}$ has countable codimension in $L_{1}$ and hence countable codimension in L. (Remark. Since by Artin-Schreier theorem the subgroup $H$ is infinite hence one easily sees that $H \simeq Z_{2} * Z_{2}$ and one may obtain the exact description of $\operatorname{Gal}\left(L_{1} / L_{1}^{H}\right)$.)

(c) Suppose that $L$ is a field of $p$-adic numbers. Consider the algebraic closure $L_{1}$ of $L$ and a transcendence basis $\left\{x_{\alpha}\right\}$ of $L$ over the prime subfield $Q$ of $L$. It is well known that for any integer $n$ the number of extensions of $L$ (contained in $L_{1}$ ) of degree less than $n$ is finite (see e.g. [4, Proposition 14, p. 40]). Hence for any integer $n, \operatorname{Gal}\left(L_{1} / L\right)$ contains only a finite number of closed normal subgroups of index less than $n$. Moreover $L \neq Q\left(\left\{x_{\alpha}\right\}\right)$ and $L$ is not a normal extension of $Q\left(\left\{x_{\alpha}\right\}\right)$ (since $L$ admits no nontrivial automorphism (see e.g. [3, Chapter XII, exercise 3])). Let $H_{1}$ be a conjugate of $\operatorname{Gal}\left(L_{1} / L\right)$ in $\operatorname{Gal}\left(L_{1} / Q\left(\left\{x_{\alpha}\right\}\right)\right)$ such that $H_{1} \neq \operatorname{Gal}\left(L_{1} / L\right)$. Let $H$ be the subgroup of $\operatorname{Gal}\left(L_{1} / Q\left(\left\{x_{\alpha}\right\}\right)\right)$ generated by $H_{1} \cup \operatorname{Gal}\left(L_{1} / L\right)$ and let $K=$ $L_{1}^{H}$. Then $K \subsetneq L$ and it follows from Lemmas 1 and 2 that $K$ is of countable codimension in $L$.

\section{REFERENCES}

1. N. Jacobson, Lectures in abstract algebra. Vol. III. Theory of fields and Galois theory, Van Nostrand, Princeton, N.J., 1964. MR 30 \#3087.

2. J. Knopfmacher and A. M. Sinclair, Fields with few extensions, Proc. Amer. Math. Soc. 29 (1971), 255-258. MR 43 \#191.

3. S. Lang, Algebra, Addison-Wesley, Reading, Mass., 1965. MR 33 \#5416.

4. —_ Algebraic numbers, Addison-Wesley, Reading, Mass., 1964. MR 28 \#3974.

Department of Mathematics and Mechanics, Warsaw University, Warsaw, POLAND 